\title{
DISPERSION OF RED BLOOD CELLS IN MICROCHANNELS: A CONFOCAL MICRO-PTV ASSESSMENT
}

\author{
RUI LIMA ${ }^{1,2}$, TAKUJI ISHIKAWA ${ }^{1}$, YOHSUKE IMAI ${ }^{1}$, HIROKI FUJIWARA ${ }^{1}$, MOTOHIRO \\ TAKEDA $^{1,3}$, NORIAKI MATSUKI $^{1,5}$, SHIGEO WADA $^{4}$, TAKAMI YAMAGUCHI ${ }^{1}$
}

1) Dept. Bioeng. \& Robot, G. S. Eng., Tohoku Univ., 6-6-01 Aoba, 980-8579 Sendai, Japan.

2) Dept. Mech. Tech., ESTiG, Braganca Polyt., C. Sta. Apolonia, 5301-857 Brag., Portugal.

3) Div. Surgical Oncology, Tohoku Univ., 2-1 Seiryo-machi, 980-8575 Sendai, Japan.

4) Dept. Mech. Sci. and Bioeng., G. S. Eng., Osaka Univ., 560-8531 Osaka, Japan.

5) New Industry Hatchery Centre, Tohoku Univ., 6-6-01 Aoba, 980-857, Sendai, Japan.

Key Words: Microcirculation, Dispersion coeficient, Red blood cells, Confocal micro-PIV/PTV.

\section{Introduction}

Blood in large arteries may be treated as a homogenous fluid from a macroscopic prospective. However, in reality blood is a suspension of deformable cells in viscous fluid plasma. In microcirculation, which comprises the smallest arteries and veins, the flow behavior of individual blood cells and their interactions provide the microrheological basis of flow properties of blood at a macroscopic level. Hence, in microcirculation it is fundamental to study the flow behavior of blood at cellular level. Several studies on both individual and concentrated RBCs have already been performed in the past $[1,2]$. However, all studies used conventional microscopes and also ghost cells to obtain visible trace RBCs through the microchannel. The present study is concerned in providing further insights into the microscale blood flow behavior through microchannels by applying an emerging optical technique known as confocal micro-PIV/PTV [3, 4, 5]. The technique consists of a spinning disk confocal microscope, high speed camera and a diode-pumped solid state (DPSS) laser combined with a single particle tracking (SPT) software (MtrackJ). Detailed measurements on the motions of RBCs were measured at different haematocrits (Hct) and the correspondent radial dispersion coefficient was determined.

\section{Materials and methods}

\subsection{Working fluids and microchannel}

Three working fluids were used in this study: dextran 40 (Dx40) containing about $3 \%$ (3Hct) $15 \%(15 \mathrm{Hct})$ and $35 \%$ (35Hct) of red blood cells (RBCs). The RBCs were collected from a healthy adult volunteer, centrifuged and then washed with physiological saline (PS). The washed RBCs were labeled with a fluorescent cell tracker (CM-Dil, C-7000, Molecular Probes) and then diluted with Dx40 to make up the required RBCs concentration by volume. All blood samples were stored hermetical at $4^{\circ} \mathrm{C}$ until the experiment was performed at controlled temperature of about $37^{\circ} \mathrm{C}$.

\subsection{Confocal micro-PTV experimental set-up}

The confocal micro-PTV system used in our experiment consists of an inverted microscope (IX71, Olympus) combined with a confocal scanning unit (CSU22, Yokogawa), a diode-pumped solid state (DPSS) laser (Laser Quantum Ltd) and a high-speed camera (Phantom v7.1) was connected into the outlet port of the CSU22. The microchannel was placed on the stage of the inverted microscope where the flow rate of the working fluids was kept constant by means of a syringe pump (KD Scientific Inc.). A thermo plate controller (Tokai Hit) was set to $37^{\circ} \mathrm{C}$. All the confocal images were captured with a resolution of $640 \times 480$ pixels, at a rate of $100 \mathrm{frames} / \mathrm{s}$ with an exposure time of $9.4 \mathrm{~ms}$. The recorded images were transfered to the computer and then evaluated in the Image J (NIH) [6] by using the manual tracking MTrackJ [7] plugin. As a result it was possible to track single RBCs through the middle plane of the microchannel. Deatailed information about the experimental set-up, used in the present study, has already been described previously [3].

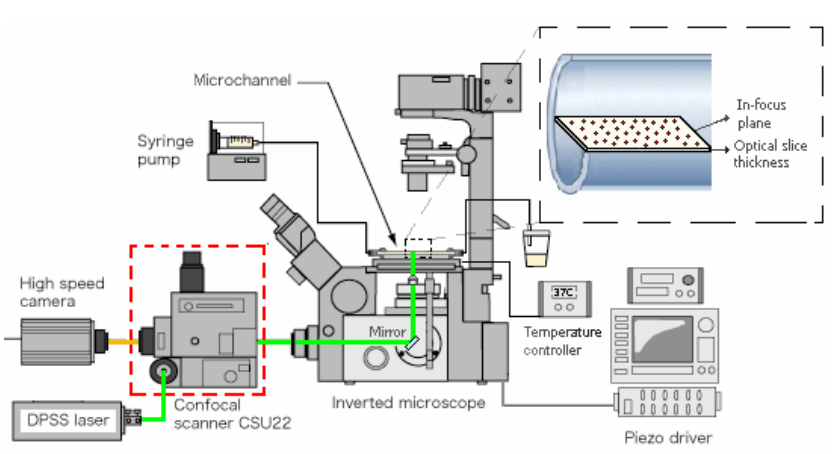

Figure 1. Experimental set-up. 


\subsection{RBC dispersion coefficient}

A $R B C$ radial dispersion coefficient $\left(D_{y y}\right)$ to quantify the radial displacement of the tracer RBCs was defined as :

$D_{y y}(t)=\frac{1}{N} \sum_{i=1}^{N} \frac{\left\langle\left(R_{i, y}(t)-R_{i, y}(0)\right)^{2}\right\rangle}{2 t}$

where $\left\langle\left(\mathrm{R}_{\mathrm{i}, \mathrm{y}}(\mathrm{t})-\mathrm{R}_{\mathrm{i}, \mathrm{y}}(0)\right)^{2}\right\rangle, \mathrm{t}$ and $\mathrm{N}$ are the mean square displacement, time interval and number of measured RBCs respectively.

\section{Results and discussion}

\subsection{Tracking displacement of RBCs at different Hcts}

By using our confocal system we were able to track single RBCs in the middle plane with Hct up to $35 \%$ (see Fig.2). Furthermore it also is possible to observe that at $3 \%$ Hct the RBC paths are almost parallel to the flow direction without any appreciable fluctuations on the radial direction. By contrast, at $15 \%$ and $35 \%$ Hct the RBC paths exhibit erratic radial displacements due the high-concentration of RBCs on the adjacent streamlines.

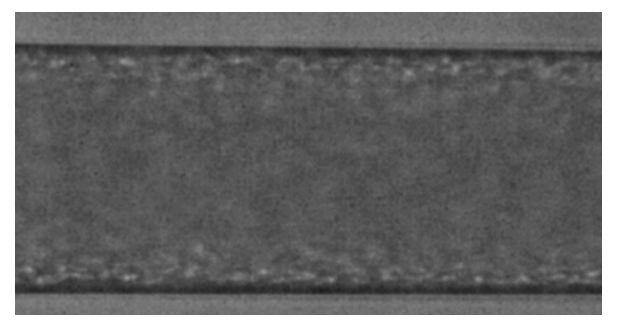

a) $35 \mathrm{Hct}$

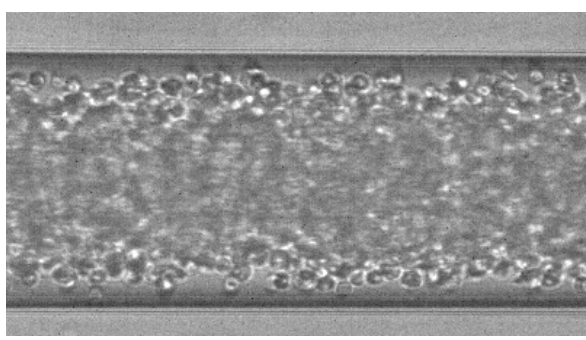

b) $15 \mathrm{Hct}$

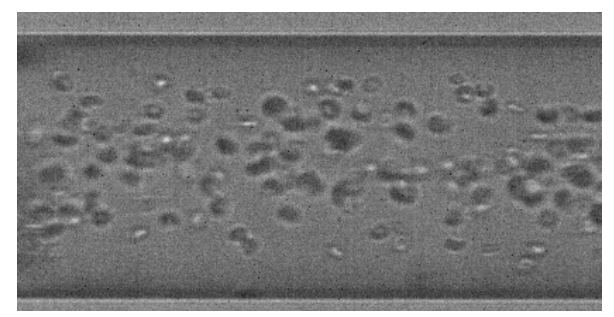

c) $3 \mathrm{Hct}$

Figure 2. Halogen images in the middle plane of a $100 \mu \mathrm{m}$ glass capillary with (a) $35 \% \mathrm{Hct}$, (b) $15 \% \mathrm{Hct}$, (c) 3\% Hct.

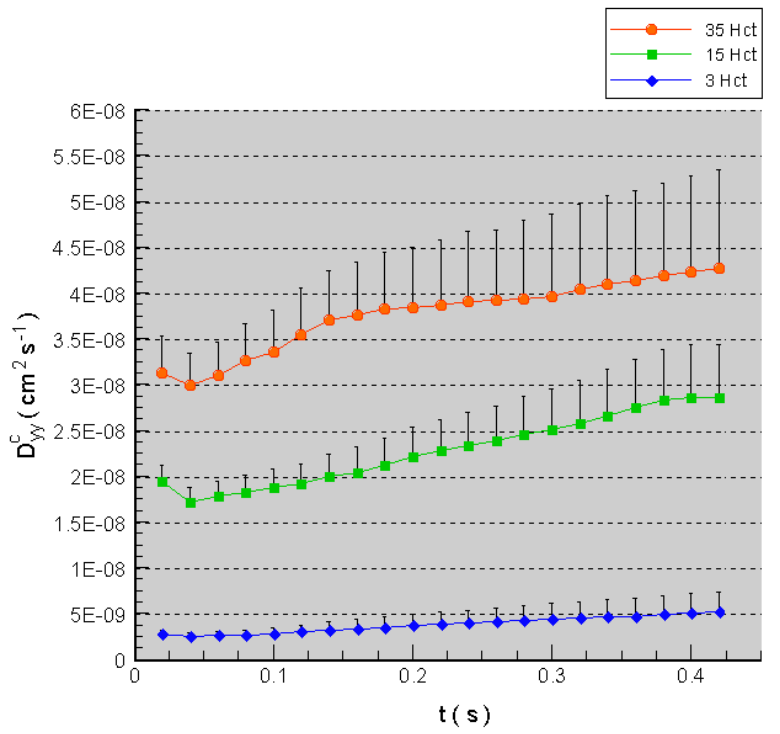

Figure 3. RBC radial dispersion coefficient $\left(D_{y y}\right)$ at the centre plane for several Hcts $(\operatorname{Re} \sim 0.005)$.

Figure 3 shows clearly that RBC radial dispersion coefficient $\left(D_{y y}\right)$ are strongly dependent on the Hct as they tend to increase with it.

\section{Acknowledgments}

This study was supported in part by the following grants: $21 \mathrm{st}$ Century COE Program for Future Medical Engineering based on Bio-nanotechnology, International Doctoral Program in Engineering from the Ministry of Education, Culture, Sports, Science and Technology of Japan (MEXT), "Revolutionary Simulation Software (RSS21)" next-generation IT program of MEXT; Grants-in-Aid for Scientific Research from MEXT and JSPS Scientific Research in Priority Areas (768) "Biomechanics at Micro- and Nanoscale Levels," Scientific Research (A) No.16200031 "Mechanism of the formation, destruction, and movement of thrombi responsible for ischemia of vital organs."

\section{References}

1) Goldsmith, H. (1971), Red cell motions and wall interactions in tube flow, Federation Proc., 30, 1578-1588.

2) Goldsmith, H. and Marlow J. (1979), Flow behavior of erythrocytes. II. Particles motions in concentrated suspensions of ghost cells, Journal of Colloid and Interf. Sci., 71, 383-407.

3) Lima, R., et al. (2006), Confocal micro-PIV measurements of three dimensional profiles of cell suspension flow in a square microchannel, Meas. Sci. Tech., 17, 797- 808.

4) Lima, R., et al. (2007), In vitro confocal micro-PIV measurements of blood flow in a square microchannel: the effect of the haematocrit on instantaneous velocity profiles, Journal of Biomechanics, 40, 2752- 2757.

5) Lima, R., et al. (2007), In vitro blood flow in a rectangular PDMS microchannel: experimental observations using a confocal micro-PIV system. Biomedical Microdevices (in press).

6) Abramoff, M., et al.. (2004), Image processing with ImageJ, Biophotonics Int., 11, 36-42.

7) Meijering, E., et al. (2006), Tracking in Molecular Bioimaging, IEEE Signal Proc. Mag., 23, 46-53. 